Relations industrielles

Industrial Relations

\title{
Le discours de Paul VI à l’O.I.T.
}

Volume 24, numéro 4, 1969

URI : https://id.erudit.org/iderudit/028075ar

DOI : https://doi.org/10.7202/028075ar

Aller au sommaire du numéro

Éditeur(s)

Département des relations industrielles de l'Université Laval

ISSN

0034-379X (imprimé)

1703-8138 (numérique)

Découvrir la revue

Citer cet article

(1969). Le discours de Paul VI à l'O.I.T. Relations industrielles / Industrial

Relations, 24(4), 796-803. https://doi.org/10.7202/028075ar

Tous droits réservés (C) Département des relations industrielles de l'Université Laval, 1969
Ce document est protégé par la loi sur le droit d'auteur. L'utilisation des services d'Érudit (y compris la reproduction) est assujettie à sa politique d'utilisation que vous pouvez consulter en ligne.

https://apropos.erudit.org/fr/usagers/politique-dutilisation/ 


\section{INFORMATIONS}

\section{Le discours de Paul VI à L'O.I.T.}

\section{RAPPELS HISTORIQUES}

\section{LE SENS DE CETTE VISITE.}

1. - C'est pour Nous un honneur et une joie de participer officiellement à cette Assemblée, à l'heure solennelle où l'Organisation Internationale du Travail célèbre le cinquantième anniversaire de sa fondation. Pourquoi sommes-Nous ici? Nous n'appartenons pas à cet organisme international. Nous sommes étranger aux questions spécifiques qui trouvent ici leurs bureaux d'étude et leurs salles de délibération, et notre mission spirituelle n'entend pas intervenir en dehors de son domaine propre. Si Nous sommes ici, c'est, Monsieur le Directeur, pour répondre à l'invitation que vous Nous avez si aimablement adressée. Et Nous sommes heureux de vous remercier publiquement, de vous dire combien Nous avons apprécié cette démarche si courtoise, combien Nous en mesurons l'importance, et de quel prix Nous apparaît sa signification.

2. - Sans compétence particulière dans les discussions techniques sur la défense et la promotion du travail humain. Nous ne sommes pourtant nullement étranger à cette grande cause du travail, qui constitue votre raison d'être, et à laquelle vous consacrez vos énergies.

\section{CHRISTIANISME ET TRAVAIL.}

3. - Dès sa première page, la Bible dont Nous sommes le messager nous présente la création comme issue du travail du Créateur et livrée au travail de la créature, dont l'effort intelligent doit la mettre en valeur, la parachever pour ainsi dire en l'humanisant, à son service. Aussi le travail est-il, selon la pensée divine, l'activité normale de l'homme, et se réjouir et jouir de ses fruits un don de Dieu, puisque chacun est tout naturellement rétribué selon ses oeuvres.

4. - A travers toutes ces pages de la Bible, le travail apparaît comme une donnée fondamentale de la condition humaine, au point que, devenu l'un de nous, le Fils de Dieu est devenu aussi en même temps un travailleur, qu'on désignait tout naturellement dans son entourage par la profession des siens: «Jésus est connu comme «le fils du charpentier». Le travail de l'homme acquérait par là les plus hautes lettres de noblesse que l'on pût imaginer et vous les avez voulu présentes à la place d'honneur, au siège de votre Organisation, par cette admirable fresque de Maurice Denis, consacrée à la dignité du travail, où le Christ apporte la Bonne Nouvelle aux travailleurs qui l'entourent, fils de Dieu eux aussi et tous frères. 


\section{RENCONTRES ENTRE L'ÉGLISE ET L'O.I.T.}

5. - S'il ne Nous appartient pas d'évoquer l'histoire qui a vu naître et s'affermir votre Organisation, Nous ne pouvons du moins passer sous silence, en ce pays hospitalier, l'oeuvre de pionniers tels que Mgr Mermillod et l'Union de Fribourg, l'admirable exemple donné par l'industriel protestant Daniel le Grand et la féconde initiative du catholique Gaspard Deeurtins, premier germe d'une conférence internationale sur le travail. Comment pourrions-Nous aussi oublier, Messieurs, que votre premier directeur avait à coeur, pour le 40 anniversaire de l'encyclique de Léon XIII sur les conditions de travail, de rendre hommage aux \& ouvriers tenaces de la justice sociale entre autres ceux qui se réclament de l'encyclique Rerum novarum ». En dressant le bilan de Dix ans d'Organisation internationale du Travail, les fonctionnaires du Bureau international du Travail n'hésitaient pas à le reconnaître: «Le grand mouvement issu, au sein de l'Église catholique, de l'encyclique Rerum novarum, a prouvé sa fécondité ».

\section{HIER . .}

6 - Le sympathie de l'Église pour votre Organisation, comme pour le monde du travail, ne cessait dès lors de se manifester et tout particulièrement dans l'encyclique Quadragesimo anno de Pie XI dans l'allocution de Pie XII au Conseil d'administration du Bureau international du Travail, dans l'encyclique Mater et magistra de Jean XXIII exprimant sa «cordiale estime envers l'O.I.T.... pour sa contribution valide et précieuse à l'instauration dans le monde, d'un ordre économique et social imprégné de justice et d'humanité, où les requêtes légitimes des travailleurs trouvent aussi leur expression ». Nous-mêmes avions la joie, au terme du Concile oecuménique du Vatican, de promulguer la constitution pastorale Gaudium et Spes élaborée par les évêques du monde entier. L'Église y réaffirme la valeur du "gigantesque effort de l'activité humaine individuelle et collective 》 tout comme la prévalence du travail des hommes sur «les autres éléments de la vie économique, qui n'ont valeur que d'instruments», avec les droits imprescriptibles et les devoirs que requiert un tel principe. Notre encyclique Populorum progressio, enfin, s'est employée à faire prendre conscience de ce que «la question sociale est devenue mondiale » avec les conséquences qui en découlent pour le développement intégral et solidaire des peuples, le développement qui est «le nouveau nom de la paix».

\section{AUJOURD'HUI . .}

7. - C'est vous le dire : Nous sommes un observateur attentif de l'oeuvre que vous accomplissez ici, bien plus, un admirateur fervent de l'activité que vous déployez un collaborateur aussi, heureux d'être invité à célébrer avec vous l'existence, les fonctions, les réalisations et les mérites de cette institution mondiale, et de le faire en ami. Et Nous n'avons garde d'oublier, en cette circonstance solennelle, les autres institutions internationales genevoises, à commencer par la Croix Rouge, toutes institutions méritantes et bien dignes d'éloges, auxquelles, Nous aimons étendre nos salutations respectueuses et nos voeux fervents.

\section{IMPORTANCE DE L'OEUVRE DÉJÀ ACCOMPLIE.}

8. - Pour Nous qui appartenons à une institution affrontée depuis deux millénaires à l'usure du temps, ces cinquante années inlassablement vouées à l'Organisation Internationale du Travail sont la source de fécondes réflexions. Chacun sait 
qu'une telle durée est un fait vraiment singulier dans l'histoire de notre siècle. La fatale précarité des choses humaines, que l'accélération de la civilisation moderne a rendue plus évidente et plus dévorante, n'a pas ébranlé votre institution, à l'idéal de laquelle Nous voulons rendre hommage : "Une paix universelle et durable ne peut être fondée que sur la base de la justice sociale». L'épreuve subie du fait de la disparition de la Société des Nations, à laquelle elle était liée organiquement, du fait aussi de la naissance de l'Organisation des Nations Unies sur un autre continent, bien loin de lui enlever ses raisons d'être, lui a au contraire fourni l'occasion, par la célèbre Déclaration de Philadelphie, voici vingt-cinq ans, de les confirmer et de les préciser, en les enracinant profondément dans la réalité du progrès de la société. "Tous les êtres humains, quels que soient leur race, leur croyance ou leur sexe, ont le droit de poursuivre leur progrès matériel et leur développement spirituel dans la liberté et la dignité, dans la sécurité économique et avec des chances égales 》.

9. - De tout coeur Nous Nous réjouissons avec vous de la vitalité de votre cinquantenaire, mais toujours jeune institution, depuis sa naissance en 1919 avec le Traité de Paix de Versailles. Qui dira les travaux, les fatigues, les veilles génératrices de tant de décisions courageuses et bénéfiques pour tous les travailleurs, comme pour la vie de l'humanité, de tous ceux qui, non sans mérite, lui ont consacré avec talent leur activité ? Entre tous, Nous ne pouvons omettre de nommer son premier directeur, Albert Thomas, et son actuel successeur, David Morse, Nous ne pouvons non plus passer sous silence le fait qu'à leur demande, et presque depuis les origines, un prêtre a toujours été au milieu de ceux qui ont constitué, construit, soutenu et servi cette insigne institution. Nous sommes reconnaissant envers tous de l'oeuvre accomplie et Nous souhaitons quelle poursuive heureusement sa mission aussi complexe que difficile, mais vraiment providentielle, pour le plus grand bien de la société moderne.

10.-Des voix mieux informées que la Nôtre diront quelle somme d'activités l'Organisation internationale du Travail a réalisée en cinquante années d'existence, et quels résultats elle a atteint avec ses cent vingt-huit conventions et ses cent trentedeux recommandations.

\section{LA VOCATION FONDAMENTALE DE L'O.I.T.}

\section{PRIMAUTÉ DE L'HOMME.}

11. - Mais comment ne pas souligner le fait primordial et d'une importance capitale que manifeste cette impressionnante documentation? Ici - et c'est un fait décisif dans l'histoire de la civilisation - ici le travail de l'homme est considéré comme digne d'une intérêt fondamental. Il n'en fut pas toujours ainsi, on le sait, dans l'histoire déjà longue de l'humanité. Que l'on songe à la conception antique du travail, au discrédit qui l'entourait, à l'esclavage qu'il entraînait, cette horrible plaie, dont il faut, hélas reconnaître qu'elle n'a pas encore entièrement disparu de la face du monde. La conception moderne, dont vous êtes les hérauts et les défenseurs, est tout autre. Elle est fondée sur un principe fondamental que le christianisme pour sa part a singulièrement mis en lumière : dans le travail, c'est l'homme qui est premier. Qu'il soit artiste ou artisan, entrepreneur, ouvrier ou paysan, manuel ou intellectuel, c'est l'homme qui travaille. C'en est donc fini de la priorité du travail sur le travailleur, et de la suprématie des exigences techniques et économiques sur les besoins humains. Jamais plus le travail au-dessus 
du travailleur, jamais plus le travail contre le travailleur, mais toujours le travail pour le travailleur, le travail au service de l'homme, de tout homme et de tout l'homme.

\section{DANS UN MONDE TECHNIQUE.}

12. - Comment l'observateur ne serait-il pas impressionné de voir que cette conception s'est précisée au moment théoriquement le moins favorable à cette affirmation du primat du facteur humain sur le produit du travail, le moment même de l'introduction progressive de la machine qui multiplie jusqu'à la démesure le rendement du travail, et tend à le remplacer ? Selon une vision abstraite des choses, le travail accompli désormais par la machine et ses énergies, fournies non plus par les bras de l'homme, mais par les formidables forces secrètes d'une nature domestiquée, aurait dû prévaloir dans l'estimation du monde moderne, jusqu'à faire oublier le travailleur, souvent libéré du poids exténuant et humiliant d'un effort physique disproportionné avec son trop faible rendement. Or, il n'en est rien. À l'heure même du triomphe de la technique et de ses effets gigantesques sur la production économique, c'est l'homme qui concentre sur lui l'attention du philosophe, du sociologue et du politique. Car il n'est en définitive de vraie richesse que de l'homme. Or, qui ne le voit, l'insertion de la technique dans le processus de l'activité humaine se ferait au détriment de l'homme, si celui-ci n'en demeurait toujours le maître, et s'il s'en dominait l'évolution. S'il « faut en toute justice reconnaître l'apport irremplaçable de l'organisation du travail et du progrès industriel à l'oeuvre du développement », vous savez mieux que quiconque les méfaits de ce qu'on a pu appeler la parcellisation du travail dans la société industrielle contemporaine. Au lieu d'aider l'homme à devenir plus homme, il le déshumanise; au lieu de l'épanouir, il l'étouffe sous une chape d'ennui pesant. Le travail demeure ambivalent, et son organisation risque de dépersonnaliser celui qui l'accomplit, si ce dernier, devenu son esclave, y abdique intelligence et liberté, jusqu'à y perdre sa dignité. Qui ne le sait, le travail, source de fruits merveilleux, quand il est véritablement créateur, peut au contraire, emporter dans le cycle de l'arbitraire, de l'injustice, de la rapacité et de la violence, devenir un véritable fléau social, comme l'attestent ces camps de travail érigés en institutions, qui ont été la honte du monde civilisé.

\section{UNE LUTTE MULTIFORME.}

13. - Qui dira le drame parfois terrible du travailleur moderne, écartelé entre son double destin de grandiose réalisateur, en proie trop souvent aux intolérables souffrances d'une condition misérable et prolétarienne, où le manque de pain se conjugue avec la dégradation sociale pour créer un état de véritable insécurité personnelle et familiale ? Vous l'avez compris. C'est le travail en tant que fait humain, premier et fondamental, qui constitue la racine vitale de votre Organisation, et en fait un arbre magnifique, un arbre qui étend ses rameaux dans le monde entier, par son caractère international, un arbre qui est un honneur pour notre temps, un arbre dont la racine toujours fertile le sollicite à une activité continue et organique. C'est cette même racine qui vous interdit de favoriser des intérêts particuliers, mais vous met au service du bien commun. C'est elle qui constitue votre génie propre et sa fécondité ; intervenir partout et toujours pour porter remède aux conflits du travail, les prévenir si possible, secourir spontanément les accidentés, élaborer de nouvelles protections contre de nouveaux dangers, améliorer le sort 
des travailleurs, en respectant l'équilibre objectif des réelles possibilités économiques, lutter contre toute ségrégation génératrice d'infériorité, pour quelque motif que ce soit - esclavage, caste, race, religion, classe - en un mot défendre, envers et contre tous, la liberté de tous les travailleurs, faire prévaloir inlassablement l'idéal de la fraternité entre les hommes, tous égaux en dignité.

\section{FAIRE PROGRESSER LA CONSCIENCE MORALE DE L'HUMANITÉ}

14. - Telle est votre vocation. Votre action ne repose, ni sur la fatalité d'une implacable lutte entre ceux qui fournissent le travail et ceux qui l'exécutent, ni sur la partialité de défenseurs d'intérêts ou de fonctions. C'est au contraire une participation organique librement organisée et socialement disciplinée aux responsabilités et aux profits du travail. Un seul but : ni l'argent, ni le pouvoir, mais le bien de l'homme. Plus qu'une conception économique, mieux qu'une conception politique, c'est une conception morale, humaine, qui vous inspire: la justice sociale à instaurer, jour après jour, librement et d'un commun accord. Découvrant toujours mieux tout ce que requiert le bien des travailleurs, vous en faites prendre peu à peu conscience et vous le proposez comme idéal. Bien plus, vous le traduisez en de nouvelles règles de comportement social, qui s'imposent comme des normes de droit. Vous assurez ainsi le passage permanent de l'ordre idéal des principes à l'ordre juridique, c'est-à-dire au droit positif. En un mot, vous affinez peu à peu, vous faites progresser la conscience morale de l'humanité. Tâche ardue et délicate certes, mais si haute et si nécessaire, qui appelle la collaboration de tous les vrais amis de l'homme. Comment ne lui apporterions-Nous pas notre adhésion et notre appui?

\section{UNE MÉTHODE : COLLABORATION ET DIALOGUE.}

15. - Sur votre route, les obstacles à écarter et les difficultés à surmonter ne manquent pas. Mais vous l'aviez prévu, et c'est pour y faire face que vous avez recours à un instrument et à une méthode qui pourraient suffire à eux seuls pour l'apologie de votre institution. Votre instrument original et organique, c'est de faire conspirer les trois forces qui sont à l'oeuvre dans la dynamique humaine du travail moderne : les hommes de gouvernement, les employeurs et les travailleurs. Et votre méthode - désormais typique paradigme - c'est d'harmoniser ces trois forces, de les faire non plus s'opposer, mais concourir « dans une collaboration courageuse et féconde», par un constant dialogue, pour l'étude et la solution de problèmes toujours renaissants et sans cesse renouvelés.

\section{UN BUT: LA PAIX UNIVERSELLE PAR LA JUSTICE SOCIALE.}

16. - Cette conception moderne et excellente est bien digne de remplacer définitivement celle qui a malheureusement dominé notre époque : conception dominée par l'efficacité recherchée à travers des agitations trop souvent génératrices de nouvelles souffrances et de nouvelles ruines, risquant ainsi d'annuler, au lieu de les consolider, les résultats obtenus au prix de luttes plus d'une fois dramatiques. Il faut le proclamer solennellement: les conflits du travail ne sauraient trouver leur remède dans des dispositions artificiellement imposées, qui privent frauduleusement le travailleur et toute la communauté sociale de leur première et inaliénable prérogative humaine, la liberté. Ils ne sauraient pas plus le trouver du reste en des situations qui résultent du seul et libre jeu - comme on dit - du déterminisme des facteurs économiques. De tels remèdes peuvent bien avoir les apparences de 
la justice, ils n'en ont point l'humaine réalité. C'est seulement en comprenant les raisons profondes de ces conflits, et en satisfaisant aux justes revendications qu'ils expriment, que vous en prévenez l'explosion dramatique et que vous en évitez les conséquences ruineuses. Avec Albert Thomas, redisons-le: «Le social» devra vaincre l'«économique». Il devra le régler et le conduire, pour mieux satisfaire à la justice ». C'est pourquoi l'Organisation Internationale du Travail apparaît aujourd'hui, dans les champs clos du monde moderne où s'affrontent dangereusement les intérêts et les idéologies, comme une voie ouverte vers un meilleur avenir de l'humanité. Plus que nulle autre institution peut-être, vous pouvez y contribuer, tout simplement en étant activement et inventivement fidèles à votre idéal : la paix universelle par la justice sociale.

17. - C'est pour cela que Nous sommes venu ici vous donner notre encouragement et notre accord, vous inviter aussi à persévérer avec ténacité dans votre mission de justice et de paix, et vous assurer de notre humble, mais sincère solidarité. Car c'est la paix du monde qui est en jeu, l'avenir de l'humanité. Cet avenir ne peut se construire que dans la paix entre toutes les familles humaines au travail, entre les classes et entre les peuples, une paix qui repose sur une justice toujours plus parfaite entre tous les hommes.

LES TACHES PRÉSENTES ET FUTURES

\section{ENTENDRE LE CRI DE L'HUMANITÉ SOUFFRANTE.}

18. - En cette heure contrastée de l'histoire de l'humanité, pleine de périls, mais remplie d'espérance, c'est à vous qu'il appartient, pour une large part, de construire la justice et, par là, d'assurer la paix. Non. Messieurs, ne croyez pas vorte oeuvre achevée, elle devient au contraire chaque jour plus urgente. Que de maux - et quels maux - que de déficiences, d'abus, d'injustices, de souffrances, que de plaintes s'élèvent encore du monde du travail. Permettez-Nous, d'être devant vous l'interprète de tous ceux qui souffrent injustement, qui sont indignement exploités, outrageusement bafoués dans leur corps et dans leur âme. avilis par un travail dégradant systématiquement voulu, organisé, imposé. Entendez ce cri de douleur qui continue à monter de l'humanité souffrante !

\section{PROCLAMER LE DROIT ET LE FAIRE RESPECTER.}

19. - Courageusement, inlassablement, luttez contre les abus toujours renaissants et les injustices sans cesse renouvelées, contraignez les intérêts particuliers à se soumettre à la vision plus large du bien commun, adaptez les anciennes dispositions aux besoins nouveaux, suscitez-en de nouvelles, engagez les nations à les ratifier, et prenez les moyens de les faire respecter car il faut le redire : «Il serait vain de proclamer des droits, si l'on ne mettait en même temps tout en oeuvre pour assurer le devoir de les respecter par tous, partout, et pour tous».

\section{DÉFENDRE L'HOMME ÉBLOUI PAR SA PROPRE ACTIVITÉ.}

20.-Osons l'ajouter: c'est contre lui-même qu'il faut défendre l'homme, l'homme menacé de n'être plus qu'une partie de lui-même, réduit, comme on dit, à une seule dimension. Il faut à tout prix l'empêcher de n'être que le pourvoyeur mécanisé d'une machine aveugle, dévoreuse du meilleur de lui-même, ou d'un État tenté d'asservir toutes les énergies à son seul service. C'est l'homme qu'il nous faut protéger, un homme emporté par les forces formidables qu'il met en oeuvre 
et comme englouti par le progrès gigantesque de son travail, un homme entraîné par l'élan irrésistible de ses inventions, et comme étourdi par le contraste croissant entre la prodigieuse augmentation des biens mis à sa disposition, et leur répartition si facilement injuste entre les hommes et entre les peuples. Le mythe de Prométhée projette son ombre inquiétante sur le drame de notre temps, où la conscience de l'homme n'arrive pas à se hausser au niveau de son activité et à assumer ses graves responsabilités, dans la fidélité au dessein d'amour de Dieu sur le monde. Aurions-nous perdu la leçon de la tragique histoire de la tour de Babel, où la conquête de la nature par l'homme oublieux de Dieu s'accompagne d'une désintégration de la société humaine.

\section{DU PLUS AVOIR AU PLUS ÊTRE: LA PARTICIPATION.}

21. - Dominant toutes les forces dissolvantes de contestation ou de babélisation, c'est la cité des hommes qu'il faut construire, une cité dont le seul ciment durable est l'amour fraternel, entre les races et les peuples, comme entre les classes et les générations. À travers les conflits qui déchirent notre temps, c'est plus qu'une revendication d'avoir, un désir légitime d'être qui s'affirme toujours davantage. Vous avez depuis cinquante ans, tissé une trame toujours plus serrée de dispositions juridiques qui protègent le travail des hommes, des femmes, des jeunes, et lui assurent une rétribution convenable. Il faut maintenant prendre les moyens d'assurer la participation organique de tous les travailleurs, non seulement aux fruits de leur travail, mais encore aux responsabilités économiques et sociales dont dépend leur avenir et celui de leurs enfants.

\section{PRIORITÉ AUX PEUPLES FAIBLES.}

22. - Il vous faut aussi assurer la participation de tous les peuples à la construction du monde, et vous préoccuper dès aujourd'hui des moins favorisés, tout comme vous aviez hier pour premier souci les catégories sociales les plus défavorisées. C'est dire que votre oeuvre législative doit se poursuivre hardiment et s'engager sur des chemins résolument nouveaux, qui assurent le droit solidaire des peuples à leur développement intégral, qui permettent singulièrement «à tous les peuples de devenir eux-mêmes les artisans de leur destin »C'est un défi qui vous est aujourd'hui lancé à l'aube de la seconde Décennie du développement. Il vous appartient de le relever. Il vous revient de prendre les décisions qui éviteront la retombée de tant d'espoirs et juguleront les tentations de la violence destructrice. Il vous faut exprimer dans des règles de droit la solidarité qui s'affirme toujours plus dans la conscience des hommes. Tout comme hier vous avez assuré par votre législation la protection et la survie du faible contre la puissance du fort - Lacordaire le disait déjà : «Entre le fort et le faible, c'est la liberté qui opprime, et la loi qui affranchit — il vous faut désormais maîtriser les droits des peuples forts, et favoriser le développement des peuples faibles en créant les conditions, non seulement théoriques, mais pratiques d'un véritable droit international du travail, à l'échelle des peuples. Comme chaque homme, chaque peuple doit pouvoir en effef, par son travail, se développer, grandir en humanité, passer de conditions moins humaines à des conditions plus humaines. Il y faut cles conditions et des moyens adaptés, une volonté commune, dont vos conventions librement élaborées entre gouvernements, travailleurs et employeurs, pourraient et devraient fournir progressivement l'expression. Plusieurs organisations spécialisées 
travaillent déjà à construire ce grand oeuvre. C'est sur cette voie qu'il vous faut progresser.

\section{ÉCOUTER LES JEUNES ET LES AIDER.}

23. - C'est dire que, si les aménagements techniques sont indispensables, il ne sauraient porter leurs fruits sans cette conscience du bien commun universel qui anime et inspire la recherche, et qui soutient l'effort, sans cet idéal qui porte les uns et les autres à se dépasser dans la construction d'un monde fraternel. Ce monde de demain, c'est aux jeunes d'aujourd'hui qu'il appartiendra de le bâtir, mais c'est à vous qu'il revient de les y préparer. Beaucoup reçoivent une formation insuffisante, n'ont pas la possibilité réelle d'apprendre un métier et de trouver un travail. Beaucoup aussi remplissent des tâches pour eux sans signification, dont la répétition monotone peut bien leur procurer un profit, mais ne suffit pas pour leur donner une raison de vivre et satisfaire leur légitime aspiration à prendre en hommes leur place dans la société. Qui ne saisit dans les pays riches, leur angoisse devant la technocratie envahissante, leur refus d'une société qui ne réussit pas à les intégrer, et dans les pays pauvres leur plainte de ne pouvoir, faute de préparation suffisante et de moyens adaptés, apporter leur concours généreux aux tâches qui les sollicitent? Dans l'actuelle mutation du monde, leur protestation retentit comme un signal de souffrance et comme un appel de justice. Au sein de la crise qui ébranle la civilisation moderne, l'attente des jeunes est anxieuse et impatiente : sachons leur ouvrir les chemins de l'avenir, leur proposer des tâches utiles et les y préparer. Il y a tant à faire en ce domaine. Vous êtes bien conscients, d'ailleurs, et Nous vous félicitons d'avoir inscrit à l'ordre du jour de votre $53^{\mathrm{e}}$ session l'étude de programmes spéciaux d'emploi et de formation de la jeunesse en vue du développement.

\section{CONCLUSION: UN COMBAT DONT LE CHRÉTIEN SAIT LE SENS}

24. - Vaste programme, Messieurs, bien digne de susciter votre enthousiasme et de galvaniser toutes vos énergies, dans le service de la grande cause qui est la vôtre - qui est aussi la nôtre - celle de l'homme. À ce combat pacifique, les disciples du Christ entendent participer de tout coeur. Car s'il importe que toutes les forces humaines collaborent pour cette promotion de l'homme, il faut mettre l'esprit à la place qui est la sienne, la première, car l'Esprit est Amour. Qui ne le voit? Cette construction dépasse les seules forces de l'homme. Mais, le chrétien le sait, il n'est pas seul avec ses frères dans cette oeuvre d'amour, de justice et de paix, où il voit la préparation et le gage de la cité éternelle qu'il attend de la grâce de Dieu. L'homme n'est pas livré à lui-même dans une foule solitaire. La cité des hommes qu'il construit est celle d'une famille de frères, d'enfants du même Père, soutenus dans leur effort par une force qui les anime et les soutient, la force de l'Esprit, force mystérieuse, mais réelle, ni magique, ni totalement étrangère à notre expérience historique et personnelle, car elle s'est exprimée en paroles humaines. Et sa voix retentit plus qu'ailleurs dans cette maison ouverte aux souffrances et aux angoisses des travailleurs, comme à ses conquêtes et à ses réalisations prestigieuses, une voix dont l'écho ineffable, aujourd'hui comme hier, ne cesse et ne cessera jamais de susciter l'espérance des hommes au travail : "Venez à moi, vous tous qui peinez et ployez sous le fardeau, et moi je vous soulagerai ». «Heureux ceux qui ont faim et soif de la justice, car ils seront rassasiés 》. 